\title{
Test performance of endobronchial ultrasound and transbronchial needle aspiration biopsy for mediastinal staging in patients with lung cancer: systematic review and meta-analysis
}

\author{
K Adams, ${ }^{1}$ P L Shah, ${ }^{2}$ L Edmonds, ${ }^{3}$ E Lim'
}

\begin{abstract}
- Appendices A and B are published online only at http:// thorax.bmj.com/content/vol64/ issueg

${ }^{1}$ Imperial College and Academic Division of Thoracic Surgery, Royal Brompton Hospital, London, UK;

${ }^{2}$ Department of Respiratory

Medicine, Royal Brompton

Hospital, London, UK; ${ }^{3}$ Library and

Knowledge Service, Papworth

Hospital, Cambridge, UK
\end{abstract}

Correspondence to:

Mr E Lim, Imperial College and

Academic Division of Thoracic

Surgery, Royal Brompton Hospital,

Sydney Street, London SW3 6NP,

UK; e.lim@rbht.nhs.uk

Received 17 October 2008

Accepted 6 May 2009

Published Online First

18 May 2009

\begin{abstract}
Background: Endobronchial ultrasound (EBUS) with transbronchial needle aspiration (TBNA) is becoming widely used for mediastinal lymph node staging in patients with known or suspected lung cancer. While a substantial number of case series have evaluated test performance of this investigation, the small sample sizes limited the ability to accurately evaluate the precision of EBUS-TBNA as a staging modality. A systematic review was performed of published studies evaluating EBUSTBNA for mediastinal lymph node staging to ascertain the pooled sensitivity and specificity of this investigation.
\end{abstract}

Methods: A literature search was constructed and performed by a professional medical librarian to identify the literature from 1960 to February 2008. Pooled specificity and sensitivity was estimated from the extracted data with an exact binomial rendition of the bivariate mixed-effects regression model.

Results: Of 365 publications, 25 were identified in which EBUS-TBNA was specifically focused on mediastinal node staging. Of these, only 10 had data suitable for extraction and analysis. The overall test performance was excellent with an area under the summary receiver operating characteristics curve of $0.99(95 \% \mathrm{Cl} 0.96$ to 1.00$)$; similarly, EBUS-TBNA had excellent pooled specificity of $1.00(95 \% \mathrm{Cl} 0.92$ to 1.00$)$ and good pooled sensitivity of $0.88(95 \% \mathrm{Cl} 0.79$ to 0.94$)$.

Conclusions: EBUS-TBNA has excellent overall test performance and specificity for mediastinal lymph node staging in patients with lung cancer. The results compare favourably with published results for computed tomography and positron emission tomography.

Diagnostic fine needle aspiration of paratracheal masses began as early as 1978, using an oesophageal biopsy needle directed via a rigid bronchoscope. ${ }^{12}$ As technology improved, smaller and more flexible aspiration needles were manufactured for use within a flexible bronchoscope. ${ }^{1}$ The development of endobronchial ultrasound (EBUS) became a significant complementary advancement. Olympus developed one of the first purpose-specific models ${ }^{3}$ with a convex probe facilitating real-time EBUS-guided transbronchial needle aspiration (TBNA) of mediastinal and hilar lymph nodes.

As technology advances, EBUS-TBNA has become increasingly available and more widely used for mediastinal lymph node staging in patients with known or suspected lung cancer. While there are a number of published case series evaluating the sensitivity and specificity of this investigation, the numbers recruited within each study are small and therefore the precision on the derived estimates in each is wide.

The aims of our study were to perform a systematic review of EBUS-TBNA and to ascertain the pooled sensitivity and specificity of this investigation compared with published results of computed tomography and positron emission tomography scans (CT and PET) for mediastinal lymph node staging in patients with lung cancer.

\section{METHODS}

\section{Study identification and eligibility}

We sought to identify all studies that used EBUS for mediastinal lymph node staging for lung cancer. The search was constructed and performed by a professional medical librarian.

A systematic literature search was performed of Medline (1950- February 2008), Embase (1974February 2008) and Cochrane Library 2008, Issue 1. To achieve the maximum sensitivity of the search strategy and identify all relevant studies, appropriate free text and thesaurus terms were used including, among others, "non-small cell lung carcinoma", "neoplasm staging" and "endobronchial" (the full search strategy is listed in online Appendix A). We searched trial registers on the Internet. In order to locate unpublished work, abstracts from major scientific meetings 2005-2007 were searched. Reference lists of all relevant studies were reviewed. No restrictions were placed on language.

Three investigators (KA, PS, EL) independently evaluated references for inclusion. Discordances were resolved by consensus. We included all studies that examined EBUS for mediastinal staging in patients with suspected or confirmed lung cancer (both small and non-small cell subtypes).

When multiple papers were published from a single institution we included all papers where there were no overlapping study periods. In the event of multiple publications with overlapping study periods, we included only one publication to prevent double counting of the patient cohorts. The selection was based on a hierarchy consisting first of patient population characteristic (ie, all patients with suspected lung cancer) followed by the publication with the largest patient series.

We excluded papers that were not about EBUS, review articles, studies that included patients with the primary underlying disease other than lung cancer, studies that were solely focused on 
Figure 1 Data and search extraction. EBUS, endobronchial ultrasound.

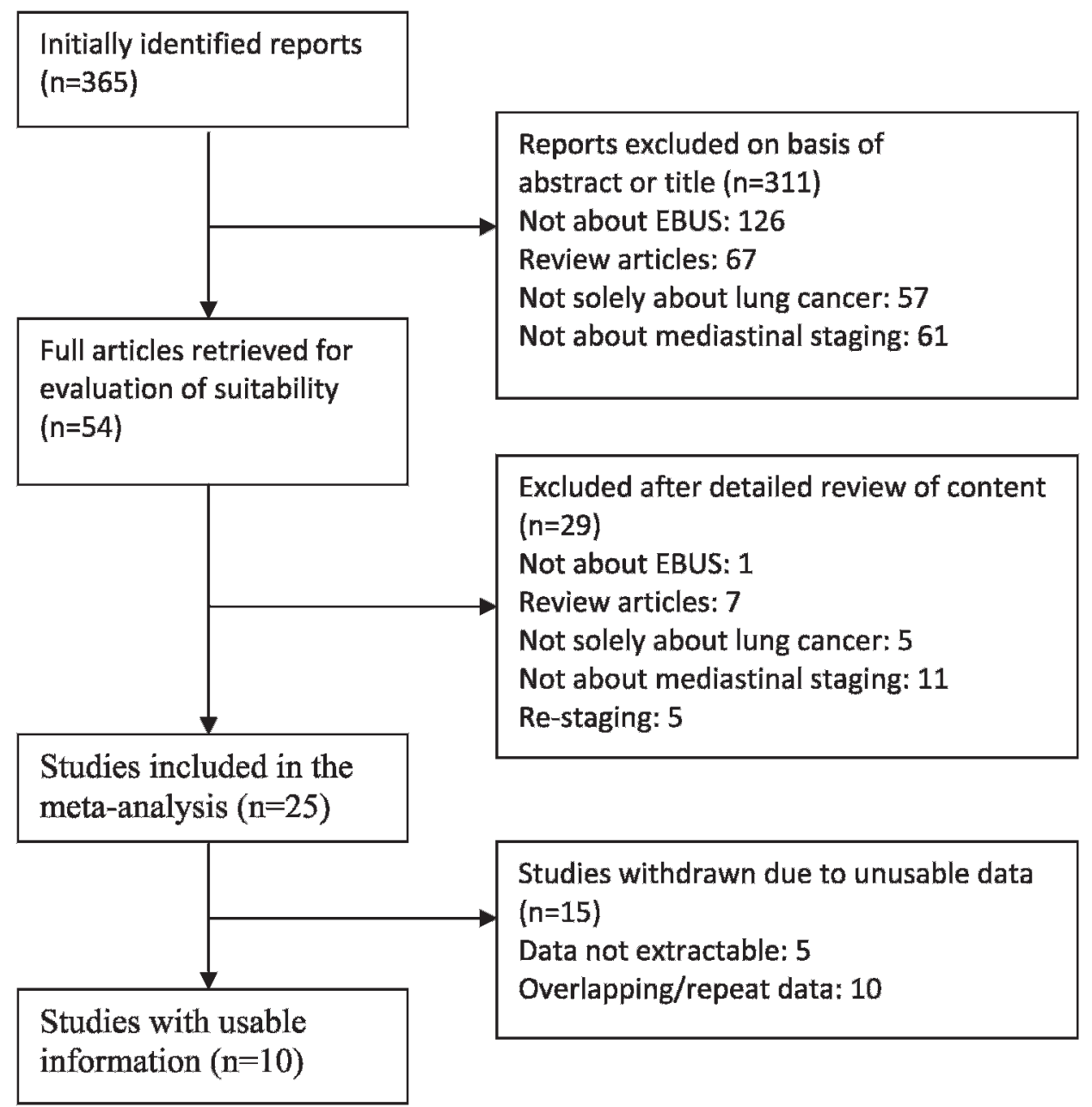

mediastinal node staging and studies of restaging after induction therapy.

\section{Quality assessment, data abstraction and synthesis (OADAS)}

The QUADAS scoring system was used to appraise the quality of all included studies. ${ }^{4}$ It is a specific tool that assesses 14 domains of design and the presentation of studies of diagnostic accuracy. Data were abstracted with the patient as the unit of analysis to prevent cluster sampling error and to focus on the ability of EBUS to accurately stage the mediastinum rather than any particular lymph node or station.

Two-by-two contingency tables were created for each study, with patients categorised into one of four options: true positive, false positive, false negative and true negative. The extracted data were pooled with an exact binomial rendition ${ }^{5}$ of the bivariate mixed-effects regression model developed by van Houwelingen $^{67}$ for treatment trial meta-analysis and modified for synthesis of diagnostic test data ${ }^{9}$ using the Midas9 module in Stata 9.2 (StataCorp, College Station, Texas, USA).

\section{RESULTS}

\section{Study selection}

Our search strategy identified 365 papers for consideration. Of these, 311 were excluded based on review of the abstracts (126 not specifically pertaining to EBUS, 67 review articles, 57 not solely about lung cancer and 61 not about mediastinal staging).

Table 1 Study characteristics

\begin{tabular}{lcll}
\hline Paper & $\begin{array}{l}\text { Eligible } \\
\text { patients (n) }\end{array}$ & Patient population & Inclusion criteria \\
\hline Okamoto $(2002)^{18}$ & 37 & Suspected lung cancer & ND \\
Yasufuku $(2005)^{15}$ & 108 & Known/suspected lung cancer & CT mediastinal lymph nodes $>1 \mathrm{~cm}$ on short axis \\
Rintoul $(2005)^{16}$ & 20 & Known/suspected lung cancer & CT mediastinal lymph nodes $>1 \mathrm{~cm}$ on short axis \\
Yasufuku $(2006)^{17}$ & 102 & Known/suspected lung cancer & Stage I-Illa \\
Plat $(2006)^{10}$ & 33 & Suspected lung cancer & PET positive mediastinal lymph nodes \\
Pierard $(2006)^{19}$ & 51 & Suspected lung cancer & PET positive mediastinal lymph nodes \\
Herth $(2006)^{13}$ & 100 & Known NSCLC & CT mediastinal lymph nodes $<1$ cm on short axis \\
Yasufuku $(2007)^{12}$ & 45 & Known/suspected lung cancer & Operable disease \\
Skwarski $(2007)^{14}$ & 300 & Known/suspected lung cancer & ND \\
Annema $(2007)^{11}$ & 21 & Known NSCLC & ND \\
\hline
\end{tabular}

\footnotetext{
ND, not described; NSCLC, non-small cell lung cancer; PET, positron emission tomography.
} 


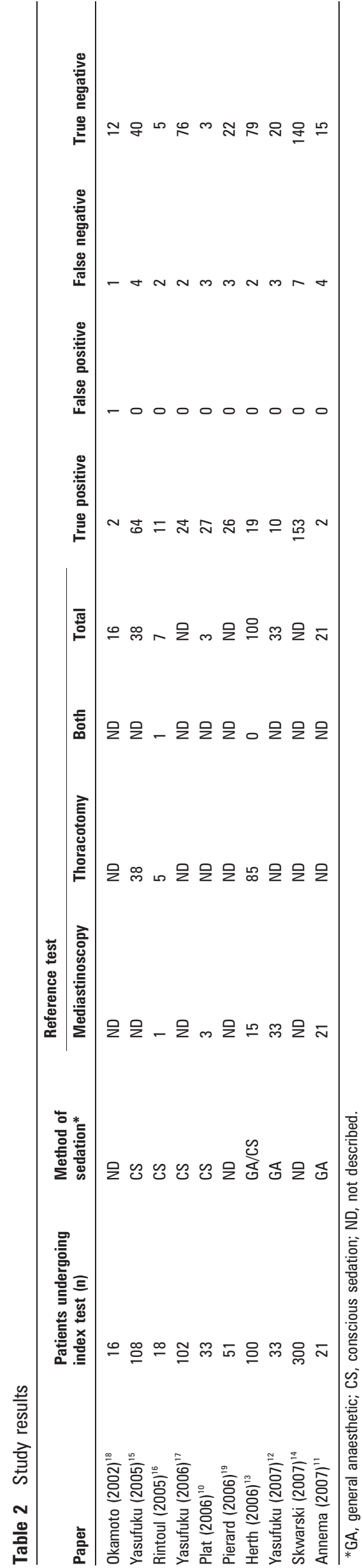

Of the 54 publications that remained, full manuscripts were obtained and a further 29 studies were excluded (1 not specifically pertaining to EBUS, 7 review articles, 5 not solely about lung cancer, 11 not about mediastinal staging and 5 that involved restaging. From the 25 publications deemed eligible for inclusion, 10 were excluded for repeat data and 5 because insufficient information was provided for data extraction (fig 1). Finally, data were extracted from 10 publications, ${ }^{10-19}$ of which 6 were full papers ${ }^{10} 1315-18$ and 4 were published abstracts ${ }^{11} 121419$ (tables 1 and 2). The details are listed in online Appendix B.

\section{Quality assessment}

Our application of the QUADAS tool revealed that there were generally low scores in all of the eligible papers. The highest score was only 9 out of a possible 14, the lowest was only 2 , with a mean of 6.1.

\section{Test performance}

The point specificity for the pooled data was 1.00 ( $95 \%$ confidence interval (CI) 0.92 to 1.00, fig 2). Unlike tests of imaging compared with tissue diagnosis, EBUS-TBNA is a test that provides tissue, similar to the reference standard. It is therefore not surprising that the pooled specificity is 1.00 (all patients who are truly negative would not be expected to test positive on EBUS). However, it is important to note that, in one case, ${ }^{18}$ although EBUS-TBNA yielded a positive result, the reference standard was negative, accounting for the lower bound of the confidence interval of 0.92 .

The point sensitivity for the pooled data was good at 0.88 ( $95 \%$ CI 0.79 to 0.94 , fig 3 ) and the area under the summary receiver operating characteristics curve was 0.99 (95\% CI 0.96 to 1.00 , fig 4).

The results corresponded to a positive likelihood ratio of 680.86 (9.66 to 48000.00) and a negative likelihood ratio of 0.12 (0.06 to 0.21$)$.

\section{DISCUSSION}

The results of our study indicate excellent overall test performance as assessed by the area under the receiver operating characteristics curve. In addition, EBUS-TBNA had excellent specificity and good sensitivity. The pooled results of specificity of EBUS-TBNA (100\%) compared favourably with a previously published meta-analysis that reported an overall specificity for CT of $79 \%$ and PET of $90 \%$. The sensitivity was also favourable at $88 \%$ in comparison with reported pooled results for CT of $59 \%$ and PET of $81 \% .{ }^{20}$

The favourable results of EBUS-TBNA compared with both CT and PET does not necessarily mean that it should be adopted as the investigation of choice for staging of mediastinal lymph nodes in patients with known or suspected lung cancer. Unlike diagnostic imaging techniques, EBUS-TBNA acquires tissue samples and is more invasive. There has been a report of torrential haemorrhage due to inadvertent biopsy of the pulmonary artery. ${ }^{21}$ In a number of the published studies, patients were admitted to hospital, anaesthetised and underwent a rigid bronchoscopy as well as EBUS-TBNA. However, this is not considered routine in clinical practice where the procedure is generally performed as a day case procedure with conscious sedation. Moreover, EBUS-TBNA can assess all lymph node stations in close proximity to the airway such as the paratracheal and subcarinal stations. Although this metaanalysis focuses on mediastinal staging, it should be noted that the endobronchial technique is also able to sample contralateral 
Figure 2 Forest plot of specificity.

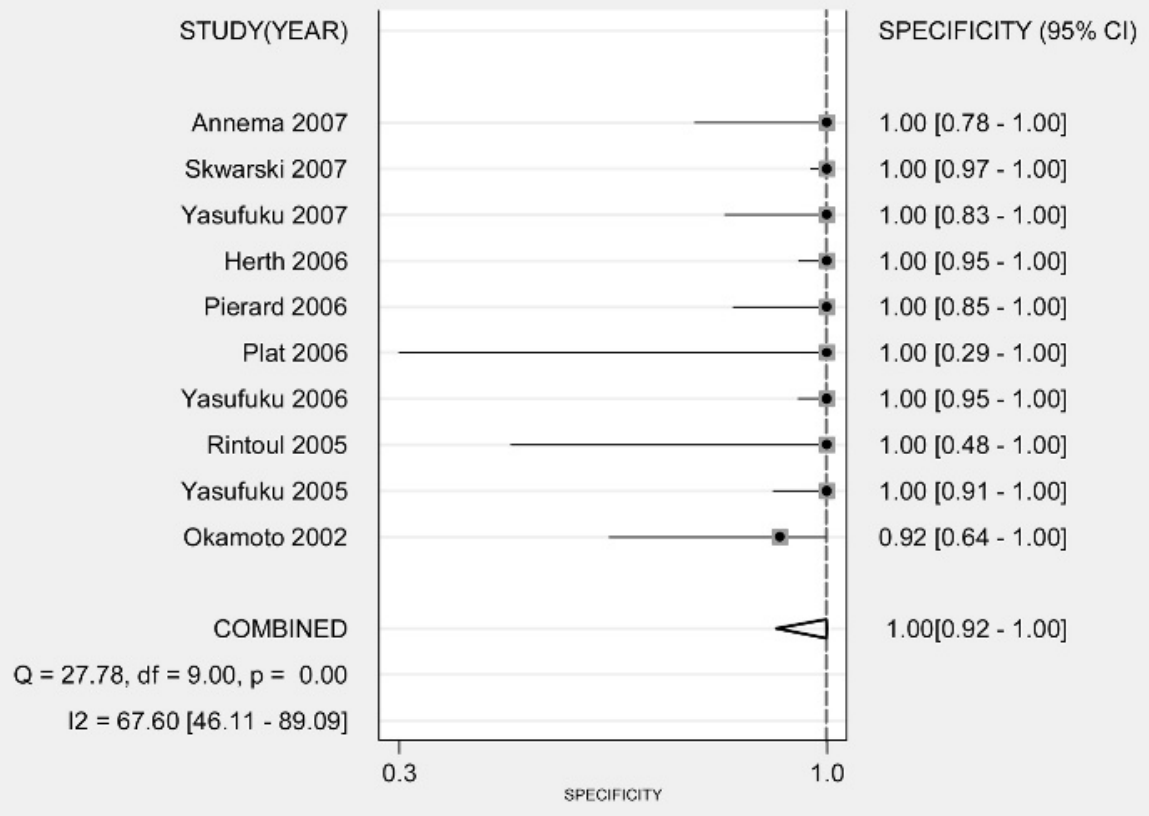

hilar lymph nodes. The main blind spots for EBUS-TBNA are the lymph nodes in the aortopulmonary window, para-aortic station, paraoesophageal stations and the inferior pulmonary ligament. In future, combination EBUS with endoscopic ultrasound (EUS) will increase the lymph nodes that are accessible using endoscopic techniques.

Integrated PET/CT carries additional advantages of screening for distant metastatic disease such as the adrenal glands and is likely to remain the initial screening investigation of choice. Surgical staging is considered the reference standard, but cervical mediastinosocpy can only sample lymph nodes in the paratracheal and subcarinal locations. Para-aortic, aortopulmonary window, paraoesophageal and inferior pulmonary ligament lymph nodes cannot be assessed unless more invasive access (mediastinotomy and video-assisted thoracoscopy) is employed.

It is likely that EBUS-TBNA will have an increasingly important role in staging the proximal mediastinal lymph node stations, especially in centres where mediastinoscopy and lymph node biopsy are not readily available or when the risks of surgical procedure are high (patients in poor health and those who have had a previous mediastinoscopy).

\section{Limitations}

There are a number of important limitations that may influence the interpretation of the results of this review. While there have

Figure 3 Forest plot of sensitivity.

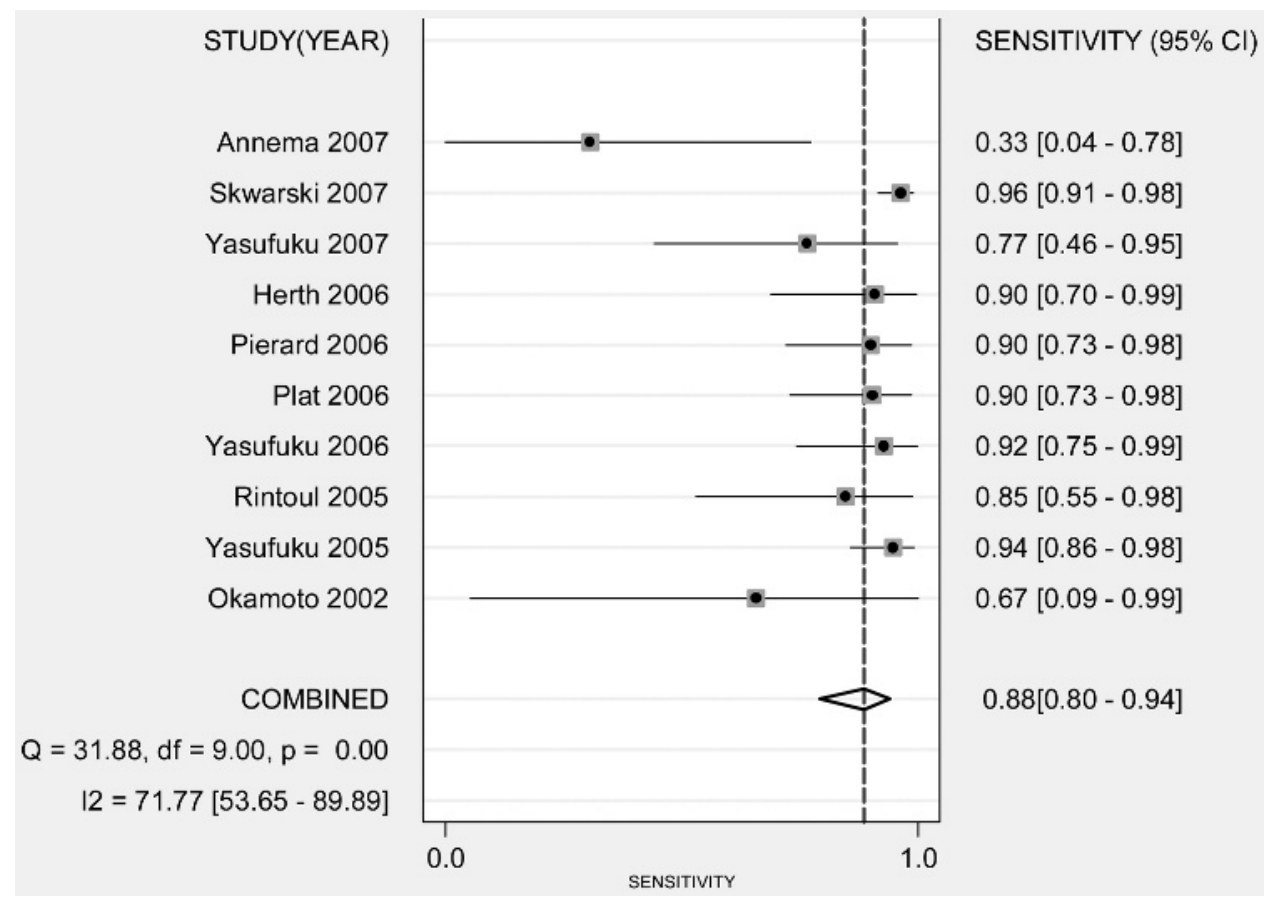


Figure 4 Summary receiver operating characteristics (SROC) plot.

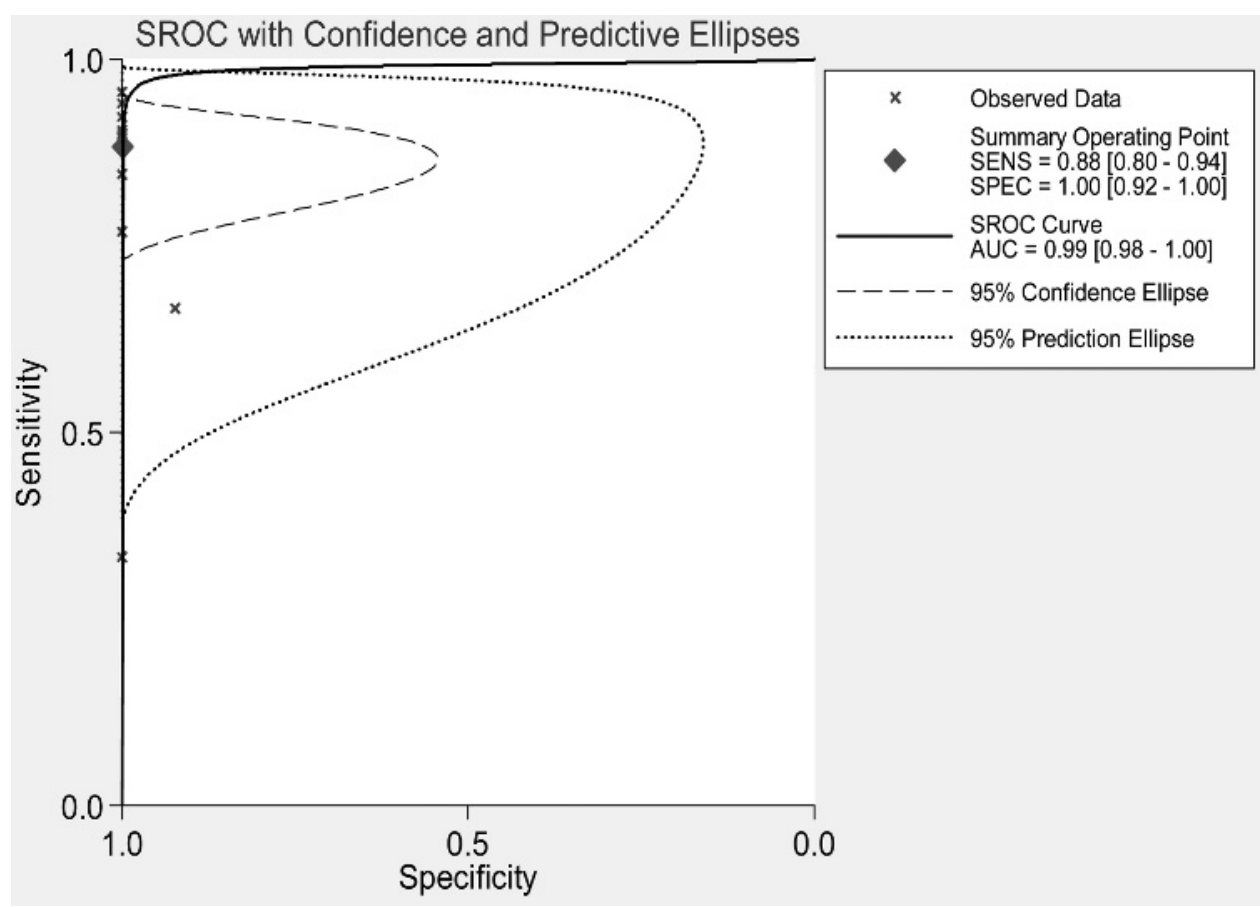

been a large number of papers published on the subject, they emanate from only a small group of authors and therefore the excellent results are generated from only a handful of experts. We would be cautious about external validity and generalising the results to less well trained practitioners and to those starting out with this technique. A degree of publication bias must be expected as it is unusual for practitioners to publish their poor results. With regard to quality assessment, in general the included studies scored poorly when assessed by QUADAS. In many studies there was poor description of reference test performance (systematic nodal dissection or mediastinoscopy staging) and, as such, we were unable to comment on the accuracy of the reference standard.

Two of the studies used radial ultrasound followed by TBNA rather than real-time ultrasound. ${ }^{10} 18$ Owing to the small numbers, it was not possible to compute and compare separate estimates for formal statistical comparisons. However, the individual study results were felt to be in keeping with the overall pooled estimates.

It would be important for future studies to directly compare the results of CT, PET-CT, EBUS-TBNA, mediastinoscopy and systematic lymph node dissection for staging and to better define where EBUS might fit into the diagnostic algorithm in relation to mediastinoscopy and lymph node biopsies. As EBUS mediastinal staging becomes more available, it should be introduced with careful auditing of each individual's results. Further studies in different institutions by different operators would greatly increase the external validity of the results of future meta-analyses on this topic.

\section{CONCLUSION}

The results of our study indicate excellent overall test performance and specificity of EBUS-TBNA when used for mediastinal lymph node staging in patients with lung cancer. In addition, the pooled test specificity and sensitivity compare favourably with published results for CT and PET. More work is required to directly compare the role of EBUS-TBNA in relation to mediastinoscopy and define its clinical utility in the era of integrated PET-CT.

Acknowledgements: The authors gratefully acknowledge the contribution of Mary Macdowell (Mount Sinai, USA) for the translation of articles published in Spanish and Tom Triebel (West Middlesex Hospital, UK) for translations of articles published in German.

Competing interests: None

Provenance and peer review: Not commissioned; externally peer reviewed.

\section{REFERENCES}

1. Herth F, Becker HD, Manegold C, et al. Endobronchial ultrasound EBUSassessment of a new diagnostic tool in bronchoscopy for staging of lung cancer Onkologie 2001;24:151-4.

2. Whyte RI. Advances in the staging of intrathoracic malignancies. World J Surg 2001:25:167-73.

3. Ernst A, Feller KD, Herth FJF. Endobronchial ultrasound in the diagnosis and staging of lung cancer and other thoracic tumors. Semin Thorac Cardiovasc Surg 2007; 19:201-5.

4. Whiting P, Rutjes AW, Reitsma JB, et al. The development of QUADAS: a tool for the quality assessmenf of studies of diagnostic accuracy included in systematic reviews. BMC Med Res Methodol 2003;3;25.

5. Chu H CS. Bivariate meta-analysis of sensitivity and specificity with sparse data: a generalized linear mixed model approach. J Clin Epidemiol 2006;59:1331-2.

6. van Houwelingen HC, Arends LR, Stijnen T. Advanced methods in meta-analysis: multivariate approach and meta-regression. Stat Med 2002;21:589-624.

7. van Houwelingen HC, Zwinderman KH, Stijnen T. A bivariate approach to metaanalysis. Stat Med 1993;12:2273-84.

8. Reitsma JB, Glas AS, Rutjes AWS, et al. Bivariate analysis of sensitivity and specificity produces informative summary measures in diagnostic reviews. J Clin Epidemiol 2005; 58:982-90.

9. Riley RDAK, Sutton AJ, Lambert P, et al. The benefits and limitations of multivariate meta-analysis, with application to diagnostic and prognostic studies. University of Leicester Medical Statistics Group Technical Report Series. Leicester: University of Leicester, 2005

10. Plat G, Pierard P, Haller A, et al. Endobronchial ultrasound and positron emission tomography positive mediastinal lymph nodes. Eur Respir J 2006;27:276-81.

11. Annema JT, Versteegh MI, Veselic M, et al. Mediastinal staging of lung cancer: EBUS-TBNA vs mediastinoscopy. Am J Respir Crit Care Med 2007;175:A619.

12. Yasufuku K, Quadri $M$, dePerrot $M$, et al. A prospective controlled trial of endobronchial ultrasound guided transbronchial needle aspiration compared to mediastinoscopy for mediastinal lymph node staging of lung cancer. Western Thoracic Surgical Association, 33rd Annual Meeting, 2007, Program and Abstracts. 
13. Herth FJF, Ernst A, Eberhardt R, et al. Endobronchial ultrasound-guided transbronchial needle aspiration of lymph nodes in the radiologically normal mediastinum. Eur Respir J 2006;28:910-4.

14. Skwarski KM, McCafferty J, Wood F, et al. Minimally invasive staging in lung cancer by real time endobronchial ultrasound FNA (EBUS). Chest 2007:132:591-2.

15. Yasufuku K, Chiyo M, Koh E, et al. Endobronchial ultrasound guided transbronchial needle aspiration for staging of lung cancer. Lung Cancer 2005;50:347-54.

16. Rintoul RC, Skwarski KM, Murchison JT, et al. Endobronchial and endoscopic ultrasound-guided real-time fine-needle aspiration for mediastinal staging. Eur Respir J 2005;25:416-21.

17. Yasufuku K, Nakajima T, Motoori K, et al. Comparison of endobronchial ultrasound, positron emission tomography, and CT for lymph node staging of lung cancer. Chest 2006:130:710-8.
18. Okamoto H, Watanabe K, Nagatomo A, et al. Endobronchial ultrasonography for mediastinal and hilar lymph node metastases of lung cancer. Chest 2002;121:1498-506.

19. Pierard PE, Faber J, Bauwens 0, et al. Evaulation of a new tool in mediastinal staging of lung cancer: real-time guided needle aspiration using endobronchial ultrasound (EBUS-NA). Chest 2006;130:146S.

20. Gould M, Kuschner WD, Rydzak C, et al. Test performance of positron emission tomography and computed tomography for mediastinal staging in patients with nonsmall-cell lung cancer. Ann Intern Med 2003;139:879-92.

21. Kanoh K, Miyazawa T, Kurimoto N, et al. Endobronchial ultrasonography guidance for transbronchial needle aspiration using a double-channel bronchoscope. Chest 2005;128:388-93.

\section{Lung alert}

\section{Asthma: a disease of the innate and adaptive immune systems?}

The paradigm that asthma is a disease solely of the adaptive immune system is gradually being challenged. This study adds weight to the suggestion that the innate immune system may play an equally pivotal role in its pathogenesis.

Using chimeric mice, the authors offer a mechanism by which exposure of the airway epithelium to house dust mite allergen might result in both forms of the immune response. Having demonstrated that the Toll-like receptor 4 (TLR4) which recognises molecular patterns found in both microbial pathogens and allergens is preferentially expressed on epithelial and dendritic cells as opposed to $\mathrm{T}$ cells, the group then show that expression of the receptor on the epithelial cell surface is the key determinant to generating activated dendritic and T helper (Th2) cells. Subsequent exposure of these TLR4-positive epithelial cells to house dust mite not only yields a rise in the Th2-associated cytokines interleukin (IL) 5 and IL13, but also generates IL25 and IL33. These cytokines are traditionally associated with an innate immune response. Furthermore, this coincides with clinical evidence of airway hyper-responsiveness, a response not seen when epithelial cells lack TLR4. Equally consistent is the fact that administration of a TLR4 receptor antagonist in the presence of house dust mite and TLR4-positive epithelial cells produces neither a clinical nor a biochemical response consistent with active airway inflammation.

Undoubtedly an exquisite piece of science, a number of questions remain. Foremost is the problem arising from previous studies which have attempted to correlate polymorphisms of the TLR4 gene locus and clinical susceptibility, only to find a highly variable relationship. Equally challenging is extrapolation of the data from mice to humans in order to identify a new therapeutic target.

- Hammad H, Chieppa M, Perros F, et al. House dust mite allergen induces asthma via Toll-like receptor 4 triggering of airway structural cells. Nat Med 2009;15:410-6.

\section{Mukhtar}

Correspondence to: Dr 0 Mukhtar, Specialist Registrar, King's College Hospital, London, UK; om1979@hotmail.com

Provenance and peer review: Not commissioned; not externally peer reviewed.

Thorax 2009;64:762. doi:10.1136/thx.2009.121004 